\title{
Characterizations to the fractional Sobolev inequality
}

\author{
Hurri-Syrjänen, Ritva
}

American Mathematical Society

2017-11

Hurri-Syrjänen, R \& Vähäkangas , A V 2017, Characterizations to the fractional Sobolev inequality . in M L Agranovsky, M Ben-Artzi , C Bénéteau , L Karp , D Khavinson, S Reich , D Shoikhet, G Weinstein \& L Zalcman (eds), Complex Analysis and Dynamical Systems VII . Contemporary Mathematics, vol. 699 , American Mathematical Society, Rhode Island, USA , pp. 145-154 , 7th International Conference on Complex Analysis and Dynamical Systems (CA \&DS VII) , Nahariya , Israel , 10/05/2015 . https://doi.org/10.1090/conm/699

http://hdl.handle.net/10138/309556

https://doi.org/10.1090/conm/699

cc_by_nc_nd

acceptedVersion

Downloaded from Helda, University of Helsinki institutional repository.

This is an electronic reprint of the original article.

This reprint may differ from the original in pagination and typographic detail.

Please cite the original version. 


\title{
CHARACTERIZATIONS TO THE FRACTIONAL SOBOLEV INEQUALITY
}

\author{
RITVA HURRI-SYRJÄNEN AND ANTTI V. VÄHÄKANGAS
}

\begin{abstract}
We characterize the fractional Sobolev inequality with fractional isocapacitary and isoperimetric inequalities. We give a sufficient condition and examples so that the fractional capacity of the closure of an open set is bounded above by the fractional perimeter of its interior.
\end{abstract}

\section{INTRODUCTION}

Let $G$ be an open set in $\mathbb{R}^{n}$ and $\delta \in(0,1)$ be given. If $1 \leq p<n / \delta$ and if there is a constant $C$ such that the inequality

$$
\left(\int_{G}|u(x)|^{n p /(n-\delta p)} d x\right)^{(n-\delta p) / n p} \leq C\left(\int_{G} \int_{G} \frac{|u(x)-u(y)|^{p}}{|x-y|^{n+\delta p}} d y d x\right)^{1 / p}
$$

holds for all measurable functions $u: G \rightarrow \mathbb{R}$ with compact support in $G$, then inequality (11) is called a fractional Sobolev inequality. In the case $p=1$ we characterize this inequality with the fractional $(\delta, 1)$-capacity $\operatorname{cap}_{\delta, 1}(\cdot, G)$ and with the fractional $\delta$-perimeter $P_{\delta}(\cdot, G)$. For the definitions we refer to Section 2 .

We state our characterization theorem.

Theorem 1.1. Suppose that $G$ is an open set in $\mathbb{R}^{n}$. Let $\delta \in(0,1)$ and a constant $C>0$ be given. Then the following conditions are equivalent.

(A) The fractional Sobolev inequality

$$
\left(\int_{G}|u(x)|^{n /(n-\delta)} d x\right)^{(n-\delta) / n} \leq C \int_{G} \int_{G} \frac{|u(x)-u(y)|}{|x-y|^{n+\delta}} d y d x
$$

holds for all measurable functions $u: G \rightarrow \mathbb{R}$ with compact support in $G$.

(B) The fractional isocapacitary inequality

$$
|K|^{(n-\delta) / n} \leq C \operatorname{cap}_{\delta, 1}(K, G)
$$

holds for every compact set $K$ in $G$.

(C) The fractional isoperimetric inequality

$$
|D|^{(n-\delta) / n} \leq 2 C P_{\delta}(D, G)
$$

holds for every open set $D \subset \subset G$ whose boundary $\partial D$ is an $(n-1)$-dimensional $C^{\infty}$-manifold in $G$.

Date: September 24, 2018.

2010 Mathematics Subject Classification. 26D10 (46E35).

Key words and phrases. Fractional Sobolev inequality, isocapacitary inequality, isoperimetric inequality. 
Theorem 1.1 is a corollary of a more general result in Section 4. We emphasize that the best constant is the same in each inequality of cases (A), (B), and (C). Our motivation has been the work of Vladimir Maz'ya on the equivalence of the classical Sobolev type inequalities and the classical isoperimetric and isocapacitary inequalities, [1], [12].

Fractional isoperimetric inequalities have been studied, for example, by Rupert L. Frank and Robert Seiringer in [6] and their stability versions by Nicola Fusco, Vincent Millot, and Massimiliano Morini in [7]. For an example of a related non-fractional case we refer to [3]. We prove that the sets $D \subset \subset G$ from case (C) are examples of the sets which satisfy the following inequality

$$
\operatorname{cap}_{\delta, 1}(\bar{D}, G) \leq 2 P_{\delta}(D, G),
$$

we refer to Section 3, Quasiballs are also examples of these sets when $G=\mathbb{R}^{n}$, Example 3.4. We give a sufficient condition for sets to satisfy inequality (2) in Theorem 3.1. We note that the left hand side of inequality (2) may be viewed as a lower bound for

$$
\left|\chi_{D}\right|_{W^{\delta, 1}(G)}=2 P_{\delta}(D, G) .
$$

Hence, this inequality is related to the question if the characteristic function $\chi_{D}$ belongs to the fractional homogeneous Sobolev space $\dot{W}^{\delta, 1}(G)$, 4].

\section{Notation AND PRELIMINARIES}

Throughout the paper we assume that $G$ is an open set in the Euclidean $n$-space $\mathbb{R}^{n}$, $n \geq 2$. The open ball centered at $x \in \mathbb{R}^{n}$ and with radius $r>0$ is $B^{n}(x, r)$. The Euclidean distance from $x \in G$ to the boundary of $G$ is written as $\operatorname{dist}(x, \partial G)$. The diameter of a set $A$ in $\mathbb{R}^{n}$ is $\operatorname{diam}(A)$. The Lebesgue $n$-measure of a measurable set $A$ is denoted by $|A|$. We write $\chi_{A}$ for the characteristic function of a set $A$.

The family $C_{0}(G)$ consists of all continuous functions $u: G \rightarrow \mathbb{R}$ with compact support in $G$. If $u: G \rightarrow \mathbb{R}$ and $t \in \mathbb{R}$ then we write shortly

$$
\{u>t\}=\{x \in G: u(x)>t\}
$$

and likewise for the sets $\{u=t\}$ and $\{u<t\}$. We let $C(*, \cdots, *)$ denote a constant which depends on the quantities appearing in the parentheses only.

Let $G$ be an open set in $\mathbb{R}^{n}$. Let $0<p<\infty$ and $0<\delta<1$ be given. We write

$$
|u|_{W^{\delta, p}(G)}=\left(\int_{G} \int_{G} \frac{|u(x)-u(y)|^{p}}{|x-y|^{n+\delta p}} d y d x\right)^{1 / p}
$$

for real-valued measurable functions $u$ on $G$. The homogeneous fractional Sobolev space $\dot{W}^{\delta, p}(G)$ consists of all measurable functions $u: G \rightarrow \mathbb{R}$ with $|u|_{W^{\delta, p}(G)}<\infty$.

The following lemma from [8, Lemma 2.6] tells that the functions $u \in \dot{W}^{\delta, p}(G)$ are locally $L^{p}$-integrable in $G$ that is, $u \in L_{\text {loc }}^{p}(G)$.

Lemma 2.1. Suppose that $G$ is an open set in $\mathbb{R}^{n}$. Let $0<p<\infty$ and $0<\delta<1$ be given. Let $K$ be a compact set in $G$. If $u \in \dot{W}^{\delta, p}(G)$, then $u \in L^{p}(K)$. 
For a compact set $K$ in $G$, its fractional $(\delta, p)$-capacity is the number

$$
\operatorname{cap}_{\delta, p}(K, G)=\inf _{u}|u|_{W^{\delta, p}(G)}^{p}
$$

where the infimum is taken over all functions $u \in C_{0}(G)$ such that $u(x) \geq 1$ for each $x \in K$.

The fractional $\delta$-perimeter of a given measurable set with respect to $G$ is defined as

$$
P_{\delta}(A, G)=\int_{A} \int_{G \backslash A} \frac{1}{|x-y|^{n+\delta}} d y d x .
$$

We note that

$$
P_{\delta}(A, G)=\frac{1}{2}\left|\chi_{A}\right|_{W^{\delta, 1}(G)} .
$$

Suppose that $A$ is a non-empty compact set in $\mathbb{R}^{n}$ and let $s \in[0, n]$. The lower $s$-dimensional Minkowski content $\mathcal{M}_{*}^{s}(A)$ is defined by

$$
\mathcal{M}_{*}^{s}(A)=\liminf _{r \rightarrow 0+} \frac{\left|A+B^{n}(0, r)\right|}{r^{n-s}} .
$$

The $s$-dimensional Hausdorff measure of $A$ is written as $\mathcal{H}^{s}(A)$. We recall from [10, p. 79] that there is a constant $C=C(s, n)>0$ such that $\mathcal{H}^{s}(A) \leq C \mathcal{M}_{*}^{s}(A)$.

Remark 2.2. Suppose that $A$ is a non-empty compact set in $\mathbb{R}^{n}$. If $0<\delta<1$ and $\delta<\rho \leq n$ are given such that $\mathcal{H}^{n-\rho}(A)<\infty$, then $\mathcal{H}^{n-\delta}(A)=0$. We refer to [10, Theorem 4.7].

We say that a closed set $\Gamma \subset G$ is an $(n-1)$-dimensional $C^{\infty}$-manifold in an open set $G$, if for each point $x \in \Gamma$ there exist open sets $U$ in $\mathbb{R}^{n-1}$ and $V$ in $\mathbb{R}$ and a smooth function $g: U \rightarrow V$ such that $x=\psi(x) \in \psi(U \times V)$ and

$$
\Gamma \cap \psi(U \times V)=\{\psi(y, g(y)): y \in U\}
$$

with a rotation $\psi$ about the point $x$.

Lemma 2.3. If $\Gamma$ is a compact $(n-1)$-dimensional $C^{\infty}$-manifold in an open set $G$ in $\mathbb{R}^{n}$, then $\mathcal{H}^{n-1}(\Gamma)<\infty$. If $u \in C_{0}^{\infty}(G)$, then

$$
\mathcal{H}^{n-1}(\{x \in G: u(x)=t\})<\infty
$$

for almost every $t>0$.

Proof. By compactness of $\Gamma$ there are open sets $U_{j}$ in $\mathbb{R}^{n-1}$ and $V_{j}$ in $\mathbb{R}$ and closed balls $B_{j}$ in $U_{j}, j=1, \ldots, N$, such that

$$
\Gamma=\bigcup_{j=1}^{N}\left\{\psi_{j}\left(y, g_{j}(y)\right): y \in B_{j}\right\}
$$

for rotations $\psi_{j}$ about points $x_{j}$ in $\Gamma$. Since the function $y \mapsto \psi_{j}\left(y, g_{j}(y)\right)$ is $L$-Lipschitz on $B_{j} \subset \subset U_{j}$ with some constant $L$, by Kirszbraun theorem on Lipschitz extension there exists an $L$-Lipschitz function $f_{j}: \mathbb{R}^{n-1} \rightarrow \mathbb{R}^{n}$ with $f_{j}(y)=\psi_{j}\left(y, g_{j}(y)\right)$ if $y \in B_{j}$. Hence, we have

$$
\mathcal{H}^{n-1}\left(f_{j}\left(B_{j}\right)\right) \leq L^{n-1} \mathcal{H}^{n-1}\left(B_{j}\right)<\infty .
$$


By subadditivity we obtain

$$
\mathcal{H}^{n-1}(\Gamma) \leq \sum_{j=1}^{N} \mathcal{H}^{n-1}\left(f_{j}\left(B_{j}\right)\right)<\infty
$$

Inequality (3) for almost every $t>0$ is a consequence of Sard's theorem [13] which, together with the implicit function theorem, implies that the level set $\{x \in G: u(x)=t\}$ is a compact $(n-1)$-dimensional $C^{\infty}$-manifold in $G$ for almost every $t>0$.

\section{ON THE FRACTIONAL CAPACITY AND PERIMETER}

Suppose that $G$ is an open set in $\mathbb{R}^{n}$ and that $0<\delta<1$ is given. Theorem 3.1 gives a suffcient condition for open sets $D \subset \subset G$ in order that the inequality

$$
\operatorname{cap}_{\delta, 1}(\bar{D}, G) \leq 2 P_{\delta}(D, G)
$$

holds. For concrete examples of the sets which satisfy inequality (4) we refer to Example 3.4.

Theorem 3.1. Suppose that $G$ is an open set in $\mathbb{R}^{n}$ and $0<\delta<1$. If $D \subset \subset G$ is an open set such that $\mathcal{H}^{n-\delta}(\partial D)=0$, then inequality (4) holds with respect to $G$.

For the proof of Theorem 3.1 we need an auxiliary result.

Lemma 3.2. Suppose that $G$ is an open set in $\mathbb{R}^{n}$ and let $0<\delta<1$ be given. Let $D \subset \subset G$ be an open set such that $\mathcal{H}^{n-\delta}(\partial D)=0$. Let $\varepsilon>0$ be given. Then, there exists a function $u$ in $C_{0}(G)$ such that $0 \leq u \leq 1$ and $u(x)=1$ for every $x \in \bar{D}$. Moreover,

$$
\int_{G \backslash D} \int_{G \backslash D} \frac{|u(x)-u(y)|}{|x-y|^{n+\delta}} d y d x<\varepsilon .
$$

Proof. We may assume that $G \neq \mathbb{R}^{n}$. If $G=\mathbb{R}^{n}$, then we just remove one point from $G \backslash \bar{D}$. Let us fix a non-negative $\psi \in C_{0}^{\infty}\left(B^{n}(0,2)\right)$ with $\psi(x)=1$ for every $x \in B^{n}(0,1)$. For a given ball $B=B^{n}\left(x_{B}, r_{B}\right)$ and $x \in \mathbb{R}^{n}$ we write $\psi^{B}(x)=\psi\left(\left(x-x_{B}\right) / r_{B}\right)$. We note that $\psi^{B}(x)=1$ for every $x \in B$ and $\psi^{B}(x)=0$ if $x \in \mathbb{R}^{n} \backslash 2 B$. By a change of variables, we find that

$$
\left|\psi^{B}\right|_{W^{\delta, 1}\left(\mathbb{R}^{n}\right)}=r_{B}^{n-\delta}|\psi|_{W^{\delta, 1}\left(\mathbb{R}^{n}\right)}<\infty .
$$

Let us fix $\varepsilon \in(0, \operatorname{dist}(\partial D, \partial G) / 6)$. Because $\mathcal{H}^{n-\delta}(\partial D)=0$, there are sets $E_{1}, E_{2}, \ldots$ in $\mathbb{R}^{n}$ such that

$$
\partial D \subset \bigcup_{i} E_{i} \quad \text { and } \quad \sum_{i} \operatorname{diam}\left(E_{i}\right)^{n-\delta}<\min \left\{\varepsilon,(\operatorname{dist}(\partial D, \partial G) / 6)^{n-\delta}\right\} .
$$

We refer to [10, Lemma 4.6].

Let $i \in \mathbb{N}$. Without loss of generality, we may assume that there is a point $x_{i} \in E_{i} \cap \partial D$. We write $B_{i}=B^{n}\left(x_{i}, 2 \operatorname{diam}\left(E_{i}\right)+2^{-i} \varepsilon\right)$. Hence, $E_{i} \subset B_{i}$. Then the family

$$
\mathcal{C}=\left\{B_{i}: i=1,2, \ldots\right\} \cup\left\{B^{n}(z, \operatorname{dist}(z, \partial D) / 3): z \in D\right\}
$$


is a covering of $\bar{D}$ with open balls. By compactness of $\bar{D}$, there is a finite subfamily $\mathcal{F} \subset \mathcal{C}$ such that $\bar{D} \subset \cup_{B \in \mathcal{F}} B$. We define $u=\min \{1, g\}$, where

$$
g=\sum_{B \in \mathcal{F}} \psi^{B}
$$

Now $u \in C_{0}(G)$ and $0 \leq u \leq 1$. Also, $u(x)=1$ for every $x \in \bar{D}$. Namely, if $x \in \bar{D}$, there exists $B_{x} \in \mathcal{F}$ such that $x \in B_{x}$ and so $g(x) \geq \psi^{B_{x}}(x)=1$. Hence, $u(x)=1$.

By definition of the function $u$

$$
\begin{aligned}
\int_{G \backslash D} \int_{G \backslash D} \frac{|u(x)-u(y)|}{|x-y|^{n+\delta}} d y d x & \leq \int_{G \backslash D} \int_{G \backslash D} \frac{|g(x)-g(y)|}{|x-y|^{n+\delta}} d y d x \\
& \leq \sum_{B \in \mathcal{F}} \int_{G \backslash D} \int_{G \backslash D} \frac{\left|\psi^{B}(x)-\psi^{B}(y)\right|}{|x-y|^{n+\delta}} d y d x .
\end{aligned}
$$

We note that $\psi^{B}(x)-\psi^{B}(y)=0$ if $x, y \in G \backslash D$ and $B=B^{n}(z$, dist $(z, \partial D) / 3)$ for some $z \in D$. Hence, by estimates in (6)

$$
\begin{aligned}
\int_{G \backslash D} \int_{G \backslash D} \frac{|u(x)-u(y)|}{|x-y|^{n+\delta}} d y d x & \leq \sum_{i=1}^{\infty} \int_{\mathbb{R}^{n}} \int_{\mathbb{R}^{n}} \frac{\left|\psi^{B_{i}}(x)-\psi^{B_{i}}(y)\right|}{|x-y|^{n+\delta}} d y d x \\
& \leq|\psi|_{W^{\delta, 1}\left(\mathbb{R}^{n}\right)} \sum_{i=1}^{\infty}\left(2 \operatorname{diam}\left(E_{i}\right)+2^{-i} \varepsilon\right)^{n-\delta} \\
& \leq C(\psi, n, \delta)\left(\varepsilon+\varepsilon^{n-\delta}\right) .
\end{aligned}
$$

The lemma is proved.

We are ready to complete the proof of Theorem 3.1 .

Proof of Theorem 3.1. Suppose that $D \subset \subset G$ is an open set and $\mathcal{H}^{n-\delta}(\partial D)=0$. Let $\varepsilon>0$ and let $u=u_{\varepsilon}$ be the $C_{0}(G)$ function given by Lemma 3.2. Then, we obtain

$$
\begin{aligned}
\operatorname{cap}_{\delta, 1}(\bar{D}, G) & \leq \int_{G} \int_{G} \frac{|u(x)-u(y)|}{|x-y|^{n+\delta}} d y d x \\
& \leq 2 \int_{D} \int_{G \backslash D} \frac{1}{|x-y|^{n+\delta}} d y d x+\int_{G \backslash D} \int_{G \backslash D} \frac{|u(x)-u(y)|}{|x-y|^{n+\delta}} d y d x \\
& <2 P_{\delta}(D, G)+\varepsilon .
\end{aligned}
$$

The theorem is proved by taking $\varepsilon \rightarrow 0$.

Corollary 3.3. Suppose that $u \in C_{0}^{\infty}(G)$. Let $0<\delta<1$ be given. Then the set

$$
D_{t}:=\{x \in G: u(x)>t\}
$$

satisfies inequality (4) with respect to $G$ for almost every $t>0$.

Proof. We note that $D_{t} \subset \subset G$ and $\partial D_{t} \subset\{x \in G: u(x)=t\}$ for every $t>0$. Hence, by Lemma 2.3, $\mathcal{H}^{n-1}\left(\partial D_{t}\right)<\infty$ for almost every $t>0$. By Remark 2.2, $\mathcal{H}^{n-\delta}\left(\partial D_{t}\right)=0$ for almost every $t>0$. Thus, the claim follows from Theorem 3.1 . 
All quasiballs satisfy inequality (44) with respect to $\mathbb{R}^{n}$.

Example 3.4. If $f: \mathbb{R}^{n} \rightarrow \mathbb{R}^{n}$ is a $K$-quasiconformal mapping [2, §3], then $D:=f\left(B^{n}(0,1)\right)$ is called a quasiball. We prove that $D$ satisfies (4) with respect to $\mathbb{R}^{n}$ for every $0<\delta<1$. Let us write

$$
r^{*}=\inf \left\{r>0: D \subset \partial D+B^{n}(0, r)\right\}<\infty .
$$

Then, by [4, Theorem 1.3] there is a constant $C=C\left(n, \delta, r^{*}, K\right)>0$ such that

$$
\int_{0}^{r^{*}}\left|\partial D+B^{n}(0, r)\right| \frac{d r}{r^{1+\delta}} \leq C\left\{|D|+\left|\chi_{D}\right|_{W^{\delta, 1}\left(\mathbb{R}^{n}\right)}\right\} .
$$

In particular, if $\mathcal{M}_{*}^{n-\delta}(\partial D)>0$, then $2 P_{\delta}\left(D, \mathbb{R}^{n}\right)=\left|\chi_{D}\right|_{W^{\delta, 1}\left(\mathbb{R}^{n}\right)}=\infty$. Hence inequality

$$
\operatorname{cap}_{\delta, 1}\left(\bar{D}, \mathbb{R}^{n}\right) \leq 2 P_{\delta}\left(D, \mathbb{R}^{n}\right)
$$

holds. On the other hand, if $\mathcal{M}_{*}^{n-\delta}(\partial D)=0$, then $\mathcal{H}^{n-\delta}(\partial D)=0$ and inequality (8) holds by Theorem 3.1. Thus, D satisfies inequality (4) with respect to $\mathbb{R}^{n}$.

\section{The MAIN RESUlT}

Theorem 1.1 is a consequence of the following, more general, result.

Theorem 4.1. Suppose that $G$ is an open set in $\mathbb{R}^{n}$. Let $1 \leq q<\infty, \delta \in(0,1)$, and a constant $C>0$ be given. Then the following conditions are equivalent.

(A) The fractional inequality

$$
\left(\int_{G}|u(x)|^{q} d x\right)^{1 / q} \leq C \int_{G} \int_{G} \frac{|u(x)-u(y)|}{|x-y|^{n+\delta}} d y d x
$$

holds for all measurable functions $u: G \rightarrow \mathbb{R}$ with compact support in $G$.

(B) The fractional isocapacitary inequality

$$
|K|^{1 / q} \leq C \operatorname{cap}_{\delta, 1}(K, G)
$$

holds for every compact set $K$ in $G$.

(C) The fractional isoperimetric inequality

$$
|D|^{1 / q} \leq 2 C P_{\delta}(D, G)
$$

holds for every open set $D \subset \subset G$ whose boundary $\partial D$ is an $(n-1)$-dimensional $C^{\infty}$-manifold in $G$.

We first give an immediate consequence of condition (A) in Theorem 4.1.

Remark 4.2. Let $G$ be an open set in $\mathbb{R}^{n}$. If $q \in[1, \infty)$ and $\delta \in(0,1)$ are given such that condition (A) in Theorem 4.1] holds with a constant $C>0$, then the inequality

$$
|A|^{1 / q} \leq 2 C P_{\delta}(A, G)
$$

holds for every measurable set $A \subset \subset G$. This follows from condition (A) when $u=\chi_{A}$. 
For the proof of Theorem 4.1 we need some auxiliary results. First we recall an extension of the classical coarea formula

$$
\int_{\mathbb{R}^{n}}|\nabla u(x)| d x=\int_{-\infty}^{\infty} \mathcal{H}^{n-1}(\{u=t\}) d t
$$

which is valid for every real-valued Lipschitz function $u$ on $\mathbb{R}^{n}$, we refer to [5, §3.2]. The following fractional coarea formula is from [1, Lemma 10].

Lemma 4.3. Suppose that $G$ is an open set in $\mathbb{R}^{n}$. Let $0<\delta<1$ be given. Then

$$
\frac{1}{2}|u|_{W^{\delta, 1}(G)}=\int_{0}^{\infty} P_{\delta}(\{u>t\}, G) d t
$$

for every $u: G \rightarrow[0, \infty)$ with $u \in \dot{W}^{\delta, 1}(G)$.

Proof. We note that

$$
|u(x)-u(y)|=\int_{0}^{\infty}\left|\chi_{\{u>t\}}(x)-\chi_{\{u>t\}}(y)\right| d t
$$

for every $x, y \in G$. On the other hand,

$$
\left|\chi_{\{u>t\}}(x)-\chi_{\{u>t\}}(y)\right|=\chi_{\{u>t\}}(x) \chi_{G \backslash\{u>t\}}(y)+\chi_{\{u>t\}}(y) \chi_{G \backslash\{u>t\}}(x) .
$$

Hence, by Fubini's theorem

$$
\begin{aligned}
|u|_{W^{\delta, 1}(G)} & =\int_{G} \int_{G} \int_{0}^{\infty} \frac{\left|\chi_{\{u>t\}}(x)-\chi_{\{u>t\}}(y)\right|}{|x-y|^{n+\delta}} d t d y d x \\
& =2 \int_{0}^{\infty} \int_{\{u>t\}} \int_{G \backslash\{u>t\}} \frac{1}{|x-y|^{n+\delta}} d x d y d t=2 \int_{0}^{\infty} P_{\delta}(\{u>t\}, G) d t .
\end{aligned}
$$

We prove an approximation lemma. Let $\varphi \in C_{0}^{\infty}\left(B^{n}(0,1)\right)$ be a non-negative bump function such that

$$
\int_{\mathbb{R}^{n}} \varphi(x) d x=1
$$

For $j \in \mathbf{N}$ and $x \in \mathbb{R}^{n}$, we write $\varphi_{j}(x)=2^{j n} \varphi(j x)$. If $u \in L^{p}\left(\mathbb{R}^{n}\right)$ and $1 \leq p<\infty$, it is well known that $u * \varphi_{j} \rightarrow u$ in $L^{p}\left(\mathbb{R}^{n}\right)$ when $j \rightarrow \infty$. We use this fact in the proof of the following lemma which tells that the standard mollification converges to $u$ in the fractional seminorm $|\cdot|_{W^{\delta, 1}(G)}$.

Lemma 4.4. Suppose that $G$ is an open set in $\mathbb{R}^{n}$. Let $0<\delta<1$ be given. Let $u: G \rightarrow \mathbb{R}$ be a function in $\dot{W}^{\delta, 1}(G)$ with compact support in $G$. Then,

$$
\left|u-u * \varphi_{j}\right|_{W^{\delta, 1}(G)} \stackrel{j \rightarrow \infty}{\longrightarrow} 0 .
$$

Proof. Let us fix $\varepsilon>0$ and let $K$ denote the support of $u$. Then $K$ is a compact set in $G$ and therefore $d=\operatorname{dist}(K, \partial G)>0$. We write

$$
\left|u-u * \varphi_{j}\right|_{W^{\delta, 1}(G)}=\int_{G} \int_{G} \frac{\left|u(x)-u * \varphi_{j}(x)-u(y)+u * \varphi_{j}(y)\right|}{|x-y|^{n+\delta}} d y d x .
$$


Since $|u|_{W^{\delta, 1}(G)}<\infty$, we may apply the monotone convergence theorem in $\mathbb{R}^{n} \times \mathbb{R}^{n}$ in order to obtain a number $\rho<d$ such that

$$
\int_{G} \int_{G \cap B^{n}(x, \rho)} \frac{|u(x)-u(y)|}{|x-y|^{n+\delta}} d y d x<\varepsilon .
$$

Now, for any $j \in \mathbf{N}$

$$
\begin{aligned}
\int_{G} \int_{G \cap B^{n}(x, \rho)} & \frac{\left|u * \varphi_{j}(x)-u * \varphi_{j}(y)\right|}{|x-y|^{n+\delta}} d y d x \\
& \leq \int_{\mathbb{R}^{n}} \varphi_{j}(z) \int_{G} \int_{G \cap B^{n}(x, \rho)} \frac{|u(x-z)-u(y-z)|}{|x-y|^{n+\delta}} d y d x d z \\
& =\int_{\mathbb{R}^{n}} \varphi_{j}(z) \int_{G-z} \int_{(G-z) \cap B^{n}(x, \rho)} \frac{|u(x)-u(y)|}{|x-y|^{n+\delta}} d y d x d z .
\end{aligned}
$$

Since $\rho<d=\operatorname{dist}(K, \partial G)=\operatorname{dist}\left(K, \mathbb{R}^{n} \backslash G\right)$, we obtain that

$$
\int_{G} \int_{G \cap B^{n}(x, \rho)} \frac{\left|u * \varphi_{j}(x)-u * \varphi_{j}(y)\right|}{|x-y|^{n+\delta}} d y d x \leq \int_{G} \int_{G \cap B^{n}(x, \rho)} \frac{|u(x)-u(y)|}{|x-y|^{n+\delta}} d y d x<\varepsilon .
$$

Hence,

$$
\int_{G} \int_{G \cap B^{n}(x, \rho)} \frac{\left|u(x)-u * \varphi_{j}(x)-u(y)+u * \varphi_{j}(y)\right|}{|x-y|^{n+\delta}} d y d x<2 \varepsilon .
$$

On the other hand, by Lemma 2.1 we have that $u \in L^{1}\left(\mathbb{R}^{n}\right)$ and therefore

$$
\begin{aligned}
& \int_{G} \int_{G \backslash B^{n}(x, \rho)} \frac{\left|u(x)-u * \varphi_{j}(x)-u(y)+u * \varphi_{j}(y)\right|}{|x-y|^{n+\delta}} d y d x \\
& \quad \leq 2\left(\int_{\mathbb{R}^{n} \backslash B^{n}(0, \rho)}|x|^{-n-\delta} d x\right) \cdot \int_{G}\left|u(x)-u * \varphi_{j}(x)\right| d x \stackrel{j \rightarrow \infty}{\longrightarrow} 0 .
\end{aligned}
$$

The claim follows by combining these estimates.

We are ready to prove Theorem 4.1.

Proof of Theorem 4.1. The implication from (A) to (B) is clear. Let us prove the implication from (B) to (C). Let $D \subset \subset G$ be an open set whose boundary $\partial D$ is an $(n-1)$-dimensional $C^{\infty}$-manifold in $G$. By condition (B), Lemma 2.3, Remark 2.2, and Theorem 3.1, we obtain that

$$
|D|^{1 / q} \leq|\bar{D}|^{1 / q} \leq C \operatorname{cap}_{\delta, 1}(\bar{D}, G) \leq 2 C P_{\delta}(D, G) .
$$

This implies condition $(\mathrm{C})$.

Let us prove the implication from (C) to (A). We fix a measurable function $u: G \rightarrow \mathbb{R}$ with compact support in $G$. Without loss of generality, we may assume that $u \in \dot{W}^{\delta, 1}(G)$. By 1-Lipschitz truncation and monotone convergence theorem, we may assume that $u$ is bounded. In particular, $u \in L^{q}(G)$. Let us write $u_{j}=|u| * \varphi_{j} \geq 0$. Since $|u| \in L^{q}(G)$ and $|u|$ has a compact support in $G$,

$$
\left(\int_{G}|u(x)|^{q} d x\right)^{1 / q}=\lim _{j \rightarrow \infty}\left(\int_{G} u_{j}(x)^{q} d x\right)^{1 / q} .
$$


We follow an argument given in [9, §7] and we focus on sufficiently large values of $j$ so that $u_{j} \in C_{0}^{\infty}(G)$. By Minkowski's integral inequality,

$$
\begin{aligned}
\left(\int_{G} u_{j}(x)^{q} d x\right)^{1 / q} & =\left(\int_{G}\left(\int_{0}^{\infty} \chi_{\left\{u_{j}>t\right\}}(x) d t\right)^{q} d x\right)^{1 / q} \\
& \leq \int_{0}^{\infty}\left(\int_{G} \chi_{\left\{u_{j}>t\right\}}(x) d x\right)^{1 / q} d t=\int_{0}^{\infty}\left|\left\{u_{j}>t\right\}\right|^{1 / q} d t .
\end{aligned}
$$

By Sard's theorem [13] for almost every $t>0$, the gradient of $u_{j}$ differs from zero at every point in the level set $\left\{x \in G: u_{j}(x)=t\right\}$. For these particular values of $t>0$, the boundary of an open set

$$
\left\{x \in G: u_{j}(x)>t\right\} \subset \subset G
$$

coincides with the level set $\left\{x \in G: u_{j}(x)=t\right\}$ and, moreover, this level set is a compact $(n-1)$-dimensional $C^{\infty}$-manifold in $G$ by the implicit function theorem.

Hence, by condition (C), Lemma 4.3, and Lemma 4.4 applied to $|u| \in \dot{W}^{\delta, 1}(G)$, we obtain

$$
\begin{aligned}
& \left(\int_{G} u_{j}(x)^{q} d x\right)^{1 / q} \\
& \leq 2 C \int_{0}^{\infty} P_{\delta}\left(\left\{u_{j}>t\right\}, G\right) d t=\frac{2 C}{2}\left|u_{j}\right|_{W^{\delta, 1}(G)} \stackrel{j \rightarrow \infty}{\longrightarrow} C|| u||_{W^{\delta, 1}(G)} \leq C|u|_{W^{\delta, 1}(G)} .
\end{aligned}
$$

Condition (A) follows.

\section{REFERENCES}

[1] Luigi Ambrosio, Guido De Philippis, and Luca Martinazzi, Gamma-convergence of nonlocal perimeter functionals, Manuscripta Math. 134 (2011), 377-403.

[2] Kari Astala, Tadeusz Iwaniec, and Gaven Martin, Elliptic Partial Differential Equations and Quasiconformal Mappings in the Plane, Princeton Mathematical Series 48, Princeton University Press, 2009.

[3] Andrea Cianchi, Nicola Fusco, Francesco Maggi, and Aldo Pratelli, On the isoperimetric deficit in Gauss space, Amer. J. Math. 133 (2011), 131-186.

[4] Daniel Faraco and Keith M. Rogers, The Sobolev norm of characteristic functions with applications to the Calderón inverse problem, Q. J. Math. 64 (2013), 133-147.

[5] Herbert Federer, Geometric Measure Theory, Springer-Verlag New York Inc., New York, 1969.

[6] Rupert L. Frank and Robert Seiringer, Non-linear ground state representations and sharp Hardy inequalities, J. Funct. Anal. 255 (2008), 3407-3430.

[7] Nicola Fusco, Vincent Millot, and Massimiliano Morini, A quantitative isoperimetric inequality for fractional perimeters, J. Funct. Anal. 261 (2011), 697-715.

[8] Ritva Hurri-Syrjänen and Antti V. Vähäkangas, Fractional Sobolev-Poincaré and fractional Hardy inequalities in unbounded John domains, preprint: http://arxiv.org/abs/1311.2863.

[9] Monika Ludwig, Anisotropic fractional perimeters, preprint: http://arxiv.org/abs/1304.0699.

[10] Pertti Mattila, Geometry of Sets and Measures in Euclidean Spaces: Fractals and Rectifiability, Cambridge Studies in Advanced Mathematics 44, Cambridge University Press, Cambridge, 1995.

[11] Vladimir Maz'ya, Lectures on isoperimetric and isocapacitary inequalities in the theory of Sobolev spaces, 307-340, Heat Kernels and Analysis on Manifolds, Graphs, and Metric Spaces (Paris , 2002), Edited by Pascal Auscher, Thierry Coulhon, and Alexander Grigor'yan, Contemp. Math. 338, Amer. Math. Soc., Providence, 2003. 
[12] Vladimir Maz'ya, Sobolev Spaces with Applications to Elliptic Partial Differential Equations, 2nd revised and augmented Edition, A Series of Comprehensive Studies in Mathematics, 342, Springer Heidelberg Dordrecht London New York, 2011.

[13] Arthur Sard, The measure of the critical values of differentiable maps, Bull. Amer. Math. Soc. 48 (1942), 883-890.

Department of Mathematics and Statistics, Gustaf Hällströmin katu 2b, Fi-00014 UniVERSity OF Helsinki, Finland

E-mail address: ritva.hurri-syrjanen@helsinki.fi

E-mail address: antti.vahakangas@helsinki.fi 